\title{
DINÂMICA DE ÍONS E ACIDIFICAÇÃO DO SOLO NOS SISTEMAS DE FERTIRRIGAÇÃO E ADUBAÇÃO SÓLIDA NA CITRICULTURA ${ }^{1}$
}

\author{
THAIS REGINA DE SOUZA², JOSÉ ANTÔNIO QUAGGIO³, GUILHERME OLIVEIRA SILVA
}

\begin{abstract}
RESUMO - A experiência em fertirrigação na citricultura mundial está localizada em clima mediterrânico, geralmente em solos alcalinos, nos quais a acidificação é apontada como uma das vantagens dessa técnica. Por outro lado, nas condições de solos tropicais, a acidificação tem sido um ponto de estrangulamento, pois poderá comprometer a sustentabilidade do sistema de fertirrigação. Este trabalho foi elaborado com objetivo de avaliar a dinâmica de íons no perfil do solo, quantificar as perdas de nutrientes por lixiviação e seus efeitos sobre a acidificação do solo em diferentes sistemas de manejo com adubação sólida e fertirrigação. O experimento foi instalado em um Argissolo VermelhoAmarelo distrófico, na cidade de Pirajuí - SP, em um talhão de variedade Natal sobre limão Cravo, com quatro anos de idade. Os tratamentos foram constituídos por duas doses de nutrientes (200; 40 e $160 \mathrm{~kg} \mathrm{ha}^{-1}$, respectivamente de $\mathrm{N}_{2} \mathrm{P}_{2} \mathrm{O}_{5}$ e $\mathrm{K}_{2} \mathrm{O}$ e sua metade) e três formas de aplicação (adubação sólida sem irrigação, adubação sólida irrigada e fertirrigação), totalizando seis tratamentos. A dinâmica de íons no solo foi monitorada pela amostragem do solo, nas profundidades de 0-20; 20-40 e 40-60 cm e também pela coleta da solução do solo, por meio de extratores de solução colocados em todas as parcelas, nas profundidades de 30 e $60 \mathrm{~cm}$, durante três anos. As maiores concentrações de íons na solução do solo ocorreram nos tratamentos fertirrigados; conseqüentemente, estes tratamentos foram os que apresentaram maiores valores de condutividade elétrica. Teores elevados de $\mathrm{N}_{-} \mathrm{NH}_{4}$ e N- $\mathrm{NO}_{3}$ na solução do solo foram observados nos tratamentos fertirrigados, nas duas profundidades, mostrando que existe grande potencial de perda de nitrogênio por lixiviação. Essas perdas de nutrientes por lixiviação, observadas nos tratamentos fertirrigados, com conseqüente acidificação do solo, podem acarretar em restrições ao uso da técnica de fertirrigação em solos tropicais.
\end{abstract}

Termos para Indexação: Citros, irrigação localizada, dinâmica de nutrientes, acidez do solo.

\section{COMPARISON OF FERTIRRIGATION AND SOLID FERTILIZATION IMPACT ON SOIL ACIDIFICATION IN CITRUS ORCHARD}

\begin{abstract}
The world-wide knowledge on citrus fertirrigation comes from the Mediterranean climate in soils originated from calcareous substrate, in which the acidification is pointed out as one of the fertirrigation advantages. On the other hand, the acidification on tropical soils has been a limiting factor, because it can compromise the fertirrigation system sustainability. Therefore, this research project was carried out in order to study the ion dynamics into the soil profile, to quantify the nutrient losses by leaching and its effects on the soil acidification on different nutritional programs, such as solid fertilization and fertirrigation. The field trial was set up in a Hapludult soil in a block of Natal sweet orange on Rangpur lime rootstock, in Pirajuí, State of Sao Paulo- Brazil, and carried out during three years. The treatments were consisted by two N, P and K rates (200-40-160 kg ha-1 and half) and three applying forms (solid fertilizers without irrigation, solid fertilizers irrigated and fertirrigation) summing up six treatments. The dynamics of ions in the soil was monitored by a soil samples taken at 0-20, 20-40 and $40-60 \mathrm{~cm}$ depth, and by soil solution samples taken, through the vacuum soil solution extractors at 30 and $60 \mathrm{~cm}$ depths. The highest ion concentrations in the soil solution occurred in the fertirrigated treatments, consequently these treatments were the ones which presented the highest electrical conductivity values. High concentration of $\mathrm{N}_{-} \mathrm{NH}_{4}$ and $\mathrm{N}-\mathrm{NO}_{3}$ was observed in the fertirrigated treatment, compared to dry fertilizer treatments, demonstrating that there is limitation to nitrification and high potential for nitrogen losses by leaching. The continuous soil bases losses, observed in the fertirrigated treatments, promoted intense soil acidification which can compromise the fertirrigation sustainability in tropical soil.
\end{abstract}

Index Terms: citrus fertirrigation, dynamic of ions, soil acidity.

\section{INTRODUÇÃO}

A área irrigada na citricultura paulista cresceu de modo acelerado nos últimos cinco anos, alcançando em 2005 cerca de 60 mil hectares, que representam cerca de $8 \%$ da área cultivada. A maior parte dessa área emprega o sistema de irrigação localizada com fertirrigação. Além do ganho em produtividade ser bastante significativo, a utilização da irrigação também melhora a qualidade dos frutos, notadamente para o mercado de fruta fresca (Duenhas et al., 2002).

Na citricultura paulista, predominam solos arenosos, nos quais a largura do bulbo úmido geralmente não ultrapassa $0,8 \mathrm{~m}$, o que provoca movimentação intensa de íons no perfil do solo e, conseqüentemente, forte acidificação, inclusive nas camadas mais profundas do solo, conforme mostram Fouché \& Bester (1986), que trabalharam durante seis anos com fertirrigação em citros e observaram decréscimo acentuado de pH. Villas Bôas et al. (1999) também alertaram para os perigos da acidificação do solo com a fertirrigação em solos tropicais. A acidificação do solo é inversamente proporcional à área de aplicação, portanto quanto mais localizada for a aplicação do fertilizante, maior será a acidificação, como no caso do gotejamento (Costa et al., 1986 e Laurindo, 2005).

A experiência em fertirrigação na citricultura mundial está localizada em clima mediterrânico, em solos originados de substrato calcário, nos quais a acidificação é apontada como uma das vantagens da fertirrigação. Por outro lado, nas condições de solos tropicais, a acidificação tem sido um ponto de estrangulamento, pois poderá comprometer a sustentabilidade do sistema de fertirrigação. Tem-se que desenvolver tecnologia própria para as condições brasileiras, pois existe pouca informação disponível na literatura nacional sobre a eficiência desse método de aplicação de fertilizantes em relação à adubação convencional sólida. Este trabalho foi elaborado com objetivo de avaliar a dinâmica de íons no perfil do solo, quantificar as perdas de nutrientes por lixiviação e seus efeitos sobre a acidificação do solo, comparando os sistemas de manejo de adubação com adubação sólida e fertirrigação.

\footnotetext{
${ }^{1}$ (Trabalho 08-2006). Recebido: 18-01-2006. Aceito para publicação: 25-08-2006.Trabalho extraído de Dissertação.

2 Mestranda do Curso de Pós-Graduação em Agricultura Tropical e Subtropical do Instituto Agronômico de Campinas Avenida Barão de Itapura 1481, CEP 13001-970, Caixa Postal 28. Bolsista FAPESP. E-mail: tr_souza@yahoo.com.br

3 Pesquisador do Instituto Agronômico de Campinas - Centro de Solos e Recursos Agroambientais - Avenida Barão de Itapura 1481, CEP 13001-970, Caixa Postal 28. E-mail: quaggio@iac.sp.gov.br

${ }^{4}$ Consultor em irrigação. Forbb, Assessoria em irrigação. E-mail: guiolisilva@techs.com.br
} 


\section{MATERIAL E MÉTODOS}

O experimento foi conduzido no período de abril de 2002 a abril de 2005 e instalado em um Argissolo Vermelho-Amarelo distrófico, na cidade de Pirajuí - SP (21º59’54" S 49²7’25" W), cujas condições edafoclimáticas a tornaram região importante de expansão da citricultura paulista. O talhão escolhido para a instalação do experimento foi o da variedade Natal sobre limão Cravo, com quatro anos de idade.

Os tratamentos foram delineados em menor número possível, mas que permitissem comparar os ganhos de eficiência da irrigação isoladamente e da fertirrigação sobre a adubação sólida convencional. Para tanto, foram empregadas duas doses de N, P e $\mathrm{K}$, definidas com base no histórico de análises de solo e folha e também em função da produtividade do talhão: a) dose anual completa, que corresponde a 200; 40 e $160 \mathrm{~kg} / \mathrm{ha}$, respectivamente de $\mathrm{N}, \mathrm{P}_{2} \mathrm{O}_{5}$ e $\mathrm{K}_{2} \mathrm{O}$, nas formas de nitrato de amônio ( $34 \%$ de N), MAP $\left(50 \%\right.$ de $\mathrm{P}_{2} \mathrm{O}_{5}$ e $10 \%$ de $\left.\mathrm{N}\right)$ e cloreto de potássio $\left(60 \%\right.$ de $\left.\mathrm{K}_{2} \mathrm{O}\right)$ tanto nos tratamentos com adubação sólida como na fertirrigação, e b) metade das doses do tratamento completo. Foram estabelecidos os seguintes tratamentos: T1- não-irrigado com adubação sólida, metade da dose; T2- não-irrigado com adubação sólida, dose completa; T3- irrigado com adubação sólida, metade da dose; T4irrigado com adubação sólida, dose completa; T5- fertirrigado, metade da dose, e T6- fertirrigado, dose completa.

As parcelas foram constituídas por três linhas com doze plantas, sendo que as dez plantas da linha central eram consideradas úteis. O espaçamento de plantio foi de 7,00 x 3,12 m do que resulta numa área total do ensaio de 1,9 ha. A análise de variância seguiu o delineamento de blocos causalizados, com seis tratamentos e quatro repetições.

$\mathrm{O}$ parcelamento da adubação sólida com $\mathrm{N}$ e $\mathrm{K}$ dos tratamentos com ou sem irrigação (T1, T2, T3 e T4) seguiu o seguinte esquema em relação às doses totais desses nutrientes: $40 \%$ na primavera (setembro a outubro); $30 \%$ no verão (dezembro a janeiro) e $30 \%$ no outono (março a abril). O fósforo foi aplicado em dose única, no mês de agosto, em todos os tratamentos com adubação sólida e também na fertirrigação.

$\mathrm{Na}$ fertirrigação, as doses de $\mathrm{N}$ e $\mathrm{K}$ foram parceladas em 40 semanas, desde o início de julho de 2002 até o final do mês de abril de 2005, em duas aplicações semanais, totalizando 80 aplicações anualmente. Esse parcelamento buscou sincronizar a adição desses nutrientes com as demandas da planta cítrica nos diferentes estádios fenológicos, definidos pela experiência dos autores.

A dinâmica de íons na solução do solo foi monitorada por extratores de solução do solo colocados em todas as parcelas, nas profundidades de 30 e $60 \mathrm{~cm}$. O extrator era constituído por um tubo de PVC, acoplado a uma cápsula de cerâmica porosa em sua extremidade inferior, na parte superior o extrator era vedado com borracha. A análise de variância dos resultados obtidos foi agrupada para a média de 9 amostragens.

As amostras de solução do solo foram trazidas para o laboratório na temperatura ambiente, eram registradas e analisadas diretamente sem filtragem ou digestão. Foram determinadas as concentrações de macronutrientes, $\mathrm{pH}$, condutividade elétrica (CE) e nitrogênio mineral $\left(\mathrm{NO}_{3}\right.$ e $\left.\mathrm{NH}_{4}\right)$, pelos métodos descritos em Raij et al. (2001). Os macronutrientes foram avaliados por leitura direta em Espectrômetro de plasma de argônio, enquanto o íon cloreto foi determinado por eletrodo seletivo em potenciômetro calibrado.

As análises de solo foram coletadas uma vez ao ano, em 2002, 2003, 2004 e 2005. Nos tratamentos sem irrigação, amostras de solo foram retiradas cerca de $0,5 \mathrm{~m}$ do interior da projeção da copa e outra cerca de 1,0 m desta em direção ao meio da rua. Essas amostras representam a maior área ocupada pelo sistema radicular das plantas. Nos tratamentos irrigados e fertirrigados, as amostras foram retiradas cerca de 0,3 m de distância da linha de emissores em direção ao meio da rua. Foram coletadas por parcela, 10 amostras simples, de forma alternada em relação ao lado da planta. Estas amostras eram homogeneizadas, dando origem à amostra composta representativa de cada parcela.

As amostras de solo coletadas foram submetidas à secagem em estufa de circulação forçada à temperatura de $40^{\circ} \mathrm{C}$ e posteriormente analisadas pelos métodos descritos por Raij et al. (2001).

Durante todo o período de coleta dos resultados, abril de 2002 a abril de 2005, foram realizadas duas aplicações de calcário no experimento, em 17-07-2002 e 18-09-2003, cujo objetivo era elevar a saturação por bases para 70\%, utilizando, respectivamente, 3,0 e 1,6 $\mathrm{t} \mathrm{ha}^{-1}$ de calcário dolomítico, com PRNT de 75\%. Essas doses de calcário foram aplicadas de forma localizada sob a projeção da copa das árvores.

As parcelas possuíam linhas duplas de gotejadores espaçadas cerca de $0,7 \mathrm{~m}$ do tronco das árvores de modo a permitir um bulbo úmido de aproximadamente $0,8 \mathrm{~m}$ de largura de cada lado da planta. O manejo da irrigação foi feito com base na medida diária da evaporação, por meio de tanque Classe A, adotando-se coeficiente de tanque Kp igual a 0,8, e o coeficiente de cultura $(\mathrm{Kc})$ variou com a fase fenológica da planta, no cálculo, respectivamente, da evapotranspiração potencial (ETP) e da evapotranspiração de cultura $(\mathrm{ETc})$. Além disso, o experimento foi monitorado por duas baterias de tensiômetros colocados nos tratamentos T2 (sem irrigação) e no tratamento T6 (fertirrigado), nas profundidades de 30; 60 e $90 \mathrm{~cm}$, procurando-se a menor flutuação possível nas medidas do potencial da água no solo, nos tensiômetros, nas profundidades de 30 e $90 \mathrm{~cm}$.

Todos os resultados obtidos foram submetidos à análise de variância (ANOVA), utilizando-se do programa de estatística Minitab versão 13 (Minitab, 2000). A diferença entre médias dos tratamentos foi avaliada através do teste de Tukey, ao nível de 5\% de probabilidade, e calculou-se a DMS (diferença mínima significativa) para cada variável que apresentou diferença entre os tratamentos com valor significativo de $1 \%$ ou $5 \%$ para o Teste F.

\section{RESULTADO E DISCUSSÃO}

\section{Dinâmica de íons na solução do solo}

As concentrações de nutrientes na solução do solo variaram com a dose e a forma de aplicação. Diferenças maiores nas concentrações de nutrientes, em função da forma de aplicação, foram observadas na profundidade de $60 \mathrm{~cm}$, sendo que, de modo geral, os tratamentos fertirrigados apresentaram as maiores concentrações, enquanto os menores valores foram observados nos tratamentos com adubação sólida irrigados (Tabela 1). Essas diferenças nas concentrações de íons, nas duas profundidades amostradas, foram mais evidentes considerando-se apenas os tratamentos irrigados. Concentrações maiores de nutrientes na profundidade de $60 \mathrm{~cm}$ demonstram maior potencial de perdas de nutrientes pela fertirrigação, mesmo quando parcelada duas vezes semanais durante 40 semanas, em relação à adubação sólida, especialmente com irrigação. Certamente esse efeito da fertirrigação está relacionado com a maior localização dos fertilizantes, a qual deverá agravar-se com o uso de apenas uma linha de gotejadores, conforme demonstrado por Laurindo (2005).

Teores muito altos de $\mathrm{N}-\mathrm{NH}_{4}^{+}$foram observados nos tratamentos fertirrigados quando comparados com os tratamentos com adubos sólidos, demonstrando que a nitrificação é prejudicada no bulbo úmido, provavelmente por limitação de arejamento, pois esse é um processo estritamente aeróbico. A nitrificação também pode ter sido afetada pela acidificação acentuada do solo, observada nestes tratamentos (Tabela 1), pois os microrganismos responsáveis pela nitrificação são sensíveis a valores baixos de $\mathrm{pH}$ (Tsai et al., 1992). Villas Bôas et al. (1999) comentaram que o amônio no solo é 
TABELA 1 - Valores de pH, condutividade elétrica e concentração de íons na solução do solo, nas profundidades de 30 e $60 \mathrm{~cm}$, para todos os tratamentos.(valores médios de 30 meses)

\begin{tabular}{|c|c|c|c|c|c|c|c|c|c|c|c|c|c|}
\hline Trat. & pH & $\mathbf{C E}$ & $\mathbf{K}$ & $\mathbf{N a}$ & $\mathbf{C a}$ & Mg & N-NH 4 & $\mathbf{H}_{2} \mathbf{P O}_{4^{-}}$ & $\mathrm{SO}^{-2}$ & $\mathrm{~N}-\mathrm{NO}_{3}$ & $\mathbf{C l}$ & $\Sigma \mathrm{C}+$ & $\Sigma \mathrm{C}-$ \\
\hline & & $\mathrm{dS} \mathrm{m}^{-1}$ & & & & & $\mathbf{m m o l}_{\mathrm{c}} \mathbf{d m}^{3}$ & & & & & & \\
\hline & & & & & & & \multicolumn{7}{|c|}{ Profundidade $30 \mathrm{~cm}$} \\
\hline T1 & $6,5 \mathrm{a}$ & $0,2 b c$ & $0,3 \mathrm{c}$ & $0,06 \mathrm{c}$ & $0,9 \mathrm{a}$ & $0,7 \mathrm{a}$ & $0,05 \mathrm{c}$ & $0,01 \mathrm{~b}$ & $0,4 \mathrm{~b}$ & $1,2 \mathrm{~b}$ & $0,4 \mathrm{~b}$ & $2,1 \mathrm{a}$ & $2,2 \mathrm{a}$ \\
\hline T2 & $5,6 \mathrm{c}$ & $0,3 \mathrm{ab}$ & $0,8 \mathrm{a}$ & $0,05 c$ & $0,8 \mathrm{ab}$ & $0,7 \mathrm{a}$ & $0,11 \mathrm{c}$ & $0,03 b$ & $0,6 \mathrm{a}$ & $1,5 \mathrm{a}$ & $0,2 \mathrm{c}$ & $2,5 \mathrm{a}$ & $2,4 \mathrm{a}$ \\
\hline T3 & $6,3 a$ & $0,2 \mathrm{c}$ & $0,4 \mathrm{c}$ & $0,07 \mathrm{c}$ & $0,4 \mathrm{c}$ & $0,3 b$ & $0,04 \mathrm{c}$ & $0,02 \mathrm{~b}$ & $0,2 \mathrm{c}$ & $0,4 \mathrm{~d}$ & $0,1 \mathrm{c}$ & $1,3 b$ & $0,9 \mathrm{c}$ \\
\hline T4 & $5,9 \mathrm{~b}$ & $0,3 \mathrm{ab}$ & $0,5 b c$ & $0,10 \mathrm{~b}$ & $0,7 \mathrm{ab}$ & $0,6 \mathrm{ab}$ & $0,29 b c$ & $0,02 b$ & $0,3 \mathrm{c}$ & $1,0 \mathrm{c}$ & $0,3 \mathrm{c}$ & $2,2 \mathrm{a}$ & $1,7 \mathrm{~b}$ \\
\hline T5 & $5,3 \mathrm{c}$ & $0,4 \mathrm{a}$ & $0,9 \mathrm{a}$ & $0,10 \mathrm{~b}$ & $0,6 \mathrm{bc}$ & $0,4 \mathrm{~b}$ & $0,68 \mathrm{a}$ & $0,07 \mathrm{a}$ & $0,1 \mathrm{~d}$ & $1,8 \mathrm{a}$ & $0,6 \mathrm{~b}$ & $2,7 a$ & $2,7 \mathrm{a}$ \\
\hline T6 & $4,9 d$ & $0,4 \mathrm{a}$ & $0,7 \mathrm{ab}$ & $0,13 \mathrm{a}$ & $0,7 \mathrm{ab}$ & $0,7 \mathrm{a}$ & $0,43 \mathrm{ab}$ & $0,03 b$ & $0,1 \mathrm{~d}$ & $1,7 \mathrm{a}$ & $1,0 \mathrm{a}$ & $2,7 \mathrm{a}$ & $2,8 \mathrm{a}$ \\
\hline \multirow[t]{2}{*}{ Tukey } & 0,5 & 0,1 & 0,2 & 0,02 & 0,2 & 0,2 & $\mathbf{0 , 3 0}$ & 0,03 & 0,1 & 0,5 & 0,2 & 0,7 & 0,7 \\
\hline & & & \multicolumn{11}{|c|}{ Profundidade $60 \mathrm{~cm}$} \\
\hline T1 & $6,9 \mathrm{a}$ & $0,3 \mathrm{bc}$ & $0,4 \mathrm{c}$ & $0,07 \mathrm{~b}$ & $1,3 \mathrm{a}$ & $0,7 \mathrm{a}$ & $0,18 \mathrm{~b}$ & $0,002 b$ & $0,4 \mathrm{a}$ & $0,7 \mathrm{c}$ & $0,6 \mathrm{~b}$ & $2,7 \mathrm{~b}$ & $2,0 \mathrm{~b}$ \\
\hline T2 & $6,8 \mathrm{a}$ & $0,4 \mathrm{bc}$ & $0,8 \mathrm{~b}$ & $0,06 \mathrm{~b}$ & $1,1 \mathrm{a}$ & $0,7 \mathrm{a}$ & $0,08 \mathrm{~b}$ & $0,003 b$ & $0,4 a$ & $0,9 \mathrm{c}$ & $0,5 b$ & $2,8 \mathrm{a}$ & $2,0 \mathrm{~b}$ \\
\hline T3 & $6,8 \mathrm{a}$ & $0,1 \mathrm{~d}$ & $0,5 \mathrm{c}$ & $0,07 b$ & $0,4 \mathrm{~b}$ & $0,3 \mathrm{~b}$ & $0,02 b$ & $0,002 b$ & $0,1 \mathrm{~b}$ & $0,3 \mathrm{c}$ & $0,1 \mathrm{~b}$ & $0,9 \mathrm{c}$ & $0,6 \mathrm{c}$ \\
\hline T4 & $6,7 \mathrm{a}$ & $0,2 \mathrm{~cd}$ & $0,3 \mathrm{c}$ & $0,07 \mathrm{~b}$ & 0,4 & $0,2 b$ & $0,09 b$ & $0,002 b$ & $0,1 b$ & $0,5 \mathrm{c}$ & $0,2 b$ & $1,1 \mathrm{c}$ & $0,9 \mathrm{c}$ \\
\hline T5 & $4,8 b$ & $0,4 \mathrm{~b}$ & $0,8 \mathrm{~b}$ & $0,09 \mathrm{~b}$ & $0,6 \mathrm{~b}$ & $0,3 \mathrm{~b}$ & $0,34 a b$ & $0,008 \mathrm{a}$ & $0,1 \mathrm{~b}$ & $2,2 b$ & $0,7 b$ & $2,2 b$ & $2,9 b$ \\
\hline T6 & $4,7 \mathrm{~b}$ & $0,8 \mathrm{a}$ & $1,3 \mathrm{a}$ & $0,14 \mathrm{a}$ & $1,1 \mathrm{a}$ & $0,5 \mathrm{ab}$ & $0,97 \mathrm{a}$ & $0,009 a$ & $0,1 b$ & $3,4 a$ & $1,4 a$ & $4,0 \mathrm{a}$ & $4,9 a$ \\
\hline Tukey & 0,4 & 0,2 & $\mathbf{0 , 3}$ & $\mathbf{0 , 0 3}$ & 0,4 & $\mathbf{0 , 3}$ & 0,66 & $\mathbf{0 , 0 0 6}$ & $\mathbf{0 , 1}$ & 0,9 & 0,5 & 1,2 & 1,4 \\
\hline
\end{tabular}

Valores seguidos pela mesma letra não diferem entre si, pelo teste de Tukey, a $5 \%$ de probabilidade.

transformado biologicamente em nitrato, após 1 a 3 semanas, com a temperatura do solo oscilando entre 25 a $30^{\circ} \mathrm{C}$. No caso da fertirrigação, essas transformações são mais demoradas, logo abaixo do emissor, local saturado com água, o que favorece a movimentação do $\mathrm{N}-\mathrm{NH}_{4}$ para camadas mais profundas do perfil do solo.

Este elevado teor de $\mathrm{N}-\mathrm{NH}_{4}$, associado às altas concentrações de $\mathrm{N}-\mathrm{NO}_{3}$ observadas nos tratamentos fertirrigados demonstram que existe grande potencial de perda de nitrogênio por lixiviação na fertirrigação, o que reafirma a importância do manejo da água para se evitar fluxo descendente no perfil do solo (Tabela 1).

Para o fósforo, que é muito reativo com a matriz coloidal do solo, a fertirrigação favoreceu também sua movimentação no perfil do solo, provavelmente em conseqüência da maior localização do $P$, e, assim, os sítios de adsorção foram saturados mais rapidamente, $\mathrm{O}$ que pode explicar os valores significativamente maiores de $\mathrm{P}$ nos tratamentos fertirrigados em relação aos demais tratamentos . Esses resultados concordam com aqueles observados por Rauschkolb et al. (1976), que verificaram movimento mais acentuado de P no solo quando aplicado via gotejamento do que com adubação sólida. Além disso, Papadopoulos (1999) mostrou que, além da maior localização dos fertilizantes, a alta frequiência da fertirrigação fosfatada pode promover o aumento no curto prazo da concentração de fósforo na solução do solo, acima do esperado, pelo comportamento do P no solo do que deve resultar maior eficiência da adubação fosfatada em relação aos adubos sólidos (Tabela 1).

O enxofre foi encontrado em menor quantidade na solução do solo nos tratamentos fertirrigados, nas duas profundidades, provavelmente devido a adsorção de sulfatos ser maior no solo quanto menor for o valor de pH (Raij, 1991). Por outro lado, maiores teores de enxofre na solução do solo foram observados nos tratamentos com adubação sólida sem irrigação, nos quais foram também observados os maiores valores de $\mathrm{pH}$.

As maiores concentrações de $\mathrm{Ca}$ e $\mathrm{Mg}$ na solução do solo, para a média das diferentes épocas, foram observadas nos tratamentos com adubação sólida. Por outro lado, no início do experimento, as maiores concentrações desses nutrientes na solução eram encontradas nos tratamentos fertirrigados, o que contribui para empobrecer a disponibilidade deles no solo e reduzir as diferenças entre os tratamentos para a média de todas as épocas. Além disso, a aplicação localizada de calcário colocou quantidades excessivas desses nutrientes sob a projeção das copas, permitindo o fornecimento contínuo, o que também diminuiu as diferenças entre os tratamentos ao longo dos anos. Essa prática da localização da calagem mostrou-se muito eficiente para reduzir os impactos negativos da acidificação acentuada do solo observada no início do experimento com a fertirrigação.

Concentrações altas de $\mathrm{Na}$ e $\mathrm{K}$ foram observadas nos tratamentos fertirrigados, demonstrando o grande potencial de perda destes cátions. Com a maior acidificação do solo provocada pela fertirrigação, ocorre menor liberação das cargas negativas dependentes de $\mathrm{pH}$ o que mantém maior quantidade dos íons $\mathrm{K}$ e Na livres na solução do solo e passíveis de serem lixiviados. É interessante observar-se que as concentrações de K na solução dos tratamentos fertirrigados são maiores do que as concentrações de $\mathrm{Ca}$ e $\mathrm{Mg}$, o que não seria esperado pela série de retenção de cátions, que em função da valência e do raio hidratado do íon, define que, o Ca é mais fortemente retido na matriz coloidal do solo que o $\mathrm{Mg}$ e, esse, mais que o K (Quaggio, 2000). Percebe-se que, nos tratamentos com adubos sólidos, tanto nos de sequeiro como nos irrigados, que essa série é mantida (Tabela 1).

\section{Balanço de cargas, condutividade elétrica e pH da solução do solo}

A despeito de inúmeras fontes de erro, desde a coleta da solução até as diferentes análises laboratoriais, o balanço de cátions e ânions foi quase perfeito na solução do solo. Isso demonstra que, na fertirrigação, a dinâmica de íons depende do equilíbrio de cargas na solução do solo, do qual depende então os processos de troca iônica, perda de nutrientes por lixiviação e a acidificação do solo, da mesma forma que no sistema de adubação sólida convencional (Tabela 1).

As perdas das bases $\mathrm{Na}, \mathrm{K}, \mathrm{Ca}, \mathrm{Mg}$ e também do íon $\mathrm{N}_{-} \mathrm{NH}_{4}$ foram contrabalanceadas pelos ânions $\mathrm{N}-\mathrm{NO}_{3} \mathrm{e} \mathrm{Cl}$. A maior perda de bases foi devida ao nitrato que, sendo absorvido em profundidade pelas raízes, contribuirá para reduzir a acidificação do solo em subsuperfície. Nota-se, porém, que o íon $\mathrm{Cl}$ nos tratamentos fertirrigados foi responsável por aproximadamente $35 \%$ da lixiviação dos cátions, ou seja, cerca de $35 \%$ da acidez gerada nesses tratamentos, foi devida ao íon $\mathrm{Cl}$ contido no cloreto de potássio. Isso mostra claramente que outras fontes de potássio, como, por exemplo, o nitrato de potássio, levaria à menor acidificação no solo, pela eliminação do íon cloreto e também por fornecer o nitrogênio na forma nítrica. 
TABELA 2 - Análise de solo realizada no ano de 2002, para a profundidade de 0-20 cm.

\begin{tabular}{|c|c|c|c|c|c|c|c|c|c|c|}
\hline Tratamento & $\begin{array}{l}\text { MO } \\
\mathrm{g} \mathrm{dm}^{-3}\end{array}$ & pH & $\begin{array}{l}P \\
\mathbf{m g ~ d m}^{-3}\end{array}$ & $\mathbf{K}$ & $\mathbf{C a}$ & $\begin{array}{l}\text { Mg } \\
\text { mmo }\end{array}$ & $\begin{array}{l}\mathbf{H}+\mathbf{A l} \\
\mathrm{dm}^{-3}\end{array}$ & SB & СТC & $\begin{array}{l}\text { V\% } \\
\%\end{array}$ \\
\hline 1 & 7,3 & $4,1 \mathrm{ab}$ & $23,3 \mathrm{~b}$ & $1,0 \mathrm{~b}$ & 4,5 & 2,8 & 20,5 & 6,8 & 27,3 & 23,8 \\
\hline 2 & 7,0 & $4,3 \mathrm{a}$ & $29,6 \mathrm{ab}$ & $1,0 \mathrm{~b}$ & 6,0 & 3,8 & 19,8 & 10,3 & 30,3 & 32,8 \\
\hline 3 & 6,8 & $4,3 \mathrm{a}$ & $22,3 \mathrm{~b}$ & $1,0 \mathrm{~b}$ & 5,0 & 2,8 & 19,5 & 9,0 & 28,5 & 31,0 \\
\hline 4 & 6,5 & $4,2 \mathrm{ab}$ & $23,0 \mathrm{~b}$ & $1,0 \mathrm{~b}$ & 5,8 & 3,8 & 21,6 & 8,0 & 29,8 & 28,3 \\
\hline 5 & 7,3 & $3,9 a b$ & $38,3 \mathrm{a}$ & $1,3 \mathrm{ab}$ & 3,8 & 2,3 & 24,3 & 5,5 & 29,8 & 19,5 \\
\hline 6 & 6,8 & $3,7 \mathrm{~b}$ & $37,5 \mathrm{a}$ & $1,6 \mathrm{a}$ & 3,0 & 1,1 & 25,0 & 4,0 & 28,8 & 13,0 \\
\hline Teste F & 0,4 & $3,7^{*}$ & $6,8^{* *}$ & $11,3 * *$ & 0,7 & 0,9 & 2,1 & 1,3 & 0,4 & 1,3 \\
\hline DMS Tukey & & 0,6 & 13,0 & 0,3 & & & & & & \\
\hline C.V. (\%) & 14,4 & 6,8 & 19,6 & 13,1 & 59,3 & 75,8 & 15,1 & 56,5 & 11,6 & 52,8 \\
\hline
\end{tabular}

Valores seguidos pela mesma letra não diferem entre si, pelo teste de Tukey, a 5\% de probabilidade.

TABELA 3 - Análise de solo realizada no ano de 2005, para as profundidades de 0-20; 20-40 e 40-60 cm.

\begin{tabular}{|c|c|c|c|c|c|c|c|c|c|c|}
\hline Tratamento & $\begin{array}{l}\text { MO } \\
\mathrm{g} \mathrm{dm}^{-3}\end{array}$ & pH & $\begin{array}{l}P \\
\text { mg dm}\end{array}$ & $\mathbf{K}$ & $\mathbf{C a}$ & $\begin{array}{l}\text { Mg } \\
\text { mmol }\end{array}$ & $\begin{array}{l}\mathbf{H}+\mathbf{A l} \\
\mathbf{d m}^{-3}\end{array}$ & SB & CTC & $\begin{array}{l}\text { V\% } \\
\%\end{array}$ \\
\hline \multicolumn{11}{|l|}{ 0-20 cm } \\
\hline $\mathbf{1}$ & 6,3 & 4,6 & 40,3 & $0,7 \mathrm{~b}$ & 11,5 & 8,5 & 22,0 & 20,7 & 42,9 & 46,5 \\
\hline 2 & 6,3 & 5,0 & 37,8 & $1,0 a b$ & 11,8 & 7,5 & 17,5 & 20,3 & 38,1 & 52,8 \\
\hline 3 & 6,8 & 4,9 & 27,8 & $0,8 \mathrm{ab}$ & 11,8 & 8,5 & 17,5 & 21,1 & 38,9 & 53,8 \\
\hline 4 & 7,0 & 4,6 & 32,8 & $0,9 \mathrm{ab}$ & 7,3 & 4,3 & 19,5 & 12,4 & 32,2 & 37,5 \\
\hline 5 & 6,0 & 4,6 & 30,0 & $1,2 \mathrm{ab}$ & 8,5 & 6,5 & 19,0 & 16,2 & 35,3 & 46,0 \\
\hline 6 & 6,8 & 4,8 & 34,5 & $1,3 \mathrm{a}$ & 10,5 & 7,8 & 19,8 & 19,6 & 39,6 & 47,8 \\
\hline Teste F & 1,4 & 1,3 & 1,4 & $2,9 *$ & 1,5 & 1,0 & 1,0 & 1,2 & 1,4 & 1,3 \\
\hline DMS Tukey & & & & 0,6 & & & & & & \\
\hline C.V. (\%) & 10,3 & 6,7 & 23,6 & 26,7 & 31,0 & 44,3 & 17,2 & 34,3 & 16,5 & 21,9 \\
\hline \multicolumn{11}{|l|}{$20-40 \mathrm{~cm}$} \\
\hline 1 & 5,3 & 4,4 & 12,0 & 0,7 & 11,5 & 9,5 & 21,8 & 21,7 & 43,6 & 46,5 \\
\hline 2 & 5,8 & 4,2 & 19,8 & 0,9 & 7,0 & 4,8 & 25,0 & 12,6 & 37,8 & 32,8 \\
\hline 3 & 6,5 & 4,5 & 11,3 & 0,6 & 7,5 & 7,0 & 19,8 & 15,1 & 35,1 & 42,3 \\
\hline 4 & 5,8 & 4,2 & 15,5 & 0,8 & 5,8 & 4,0 & 23,8 & 10,6 & 34,4 & 30,3 \\
\hline 5 & 5,8 & 4,2 & 25,0 & 1,1 & 4,5 & 2,8 & 23,5 & 8,4 & 32,2 & 26,8 \\
\hline 6 & 5,8 & 4,2 & 19,8 & 1,3 & 5,8 & 4,0 & 26,0 & 11,1 & 37,1 & 31,3 \\
\hline Teste F & $\mathbf{0 , 7}$ & 1,6 & 1,8 & 2,6 & 1,9 & 2,2 & $\mathbf{0 , 8}$ & 1,9 & 1,0 & 2,0 \\
\hline C.V. (\%) & 16,0 & 5,1 & 45,2 & 37,7 & 51,3 & 63,4 & 21,8 & 52,2 & 21,1 & 31,2 \\
\hline \multicolumn{11}{|l|}{$40-60 \mathrm{~cm}$} \\
\hline 1 & 5,3 & 4,3 & 5,5 & 0,6 & 7,3 & 5,3 & 25,5 & 13,1 & 38,8 & 34,0 \\
\hline 2 & 5,5 & 4,2 & 8,0 & 0,9 & 6,0 & 4,0 & 26,5 & 10,9 & 37,3 & 29,0 \\
\hline 3 & 5,8 & 4,1 & 5,3 & 0,6 & 6,0 & 5,3 & 30,8 & 11,8 & 42,6 & 28,8 \\
\hline 4 & 5,8 & 4,1 & 8,0 & 0,8 & 5,5 & 4,0 & 27,3 & 10,3 & 37,4 & 27,5 \\
\hline 5 & 5,5 & 4,1 & 11,0 & 1,2 & 4,8 & 3,3 & 29,8 & 9,2 & 39,0 & 25,8 \\
\hline 6 & 5,3 & 4,0 & 8,5 & 1,2 & 5,8 & 4,8 & 34,5 & 11,7 & 46,4 & 26,0 \\
\hline Teste F & $\mathbf{0 , 3}$ & $\mathbf{0 , 7}$ & 1,3 & 2,0 & $\mathbf{0 , 8}$ & $\mathbf{0 , 8}$ & 0,6 & $\mathbf{0 , 6}$ & 0,5 & 0,5 \\
\hline C.V. $(\%)$ & 14,1 & 4,5 & 49,3 & 45,0 & 30,5 & 40,7 & 30,9 & 31,8 & 25,1 & 29,9 \\
\hline
\end{tabular}

Valores seguidos pela mesma letra não diferem entre si, pelo Teste de Tukey, a $5 \%$ de probabilidade.

Na fertirrigação, é possível fazer alterações rápidas e precisas nas quantidades de nutrientes aplicados, portanto o monitoramento das concentrações desses elementos na solução do solo, por meio de medidas da condutividade elétrica, permite ajustar a demanda da planta com o fornecimento dos nutrientes, evitando-se, ainda, as perdas por lixiviação durante o ciclo da cultura (Villas Boas et al., 2005). Os resultados das Tabelas 1 e 2 mostram que existe relação direta $\left(R^{2}=0,95^{*}\right.$, dados não apresentados) entre a concentração de íons na solução do solo e condutividade elétrica (CE) nas diferentes profundidades amostradas. Souza et al. (2003) mencionam que as perdas de nutrientes por lixiviação na fertirrigação poderiam ser reduzidas monitorando-se a condutividade na solução do solo o que está em concordância com os resultados deste trabalho.

\section{Acidificação do solo}

Os resultados das análises químicas de solo no início do experimento, em 2002, no qual a análise foi realizada apenas para a profundidade de $0-20 \mathrm{~cm}$, e no final, em 2005 , encontram-se nas Tabelas 2 e 3 . As diferenças entre os tratamentos foram mais evidentes no primeiro ano de amostragem 2002. O tratamento fertirrigado T6 foi o que apresentou significativamente o menor valor de $\mathrm{pH}$ em relação aos outros tratamentos, porém o tratamento fertirrigado $\mathrm{T} 5$ também apresentou valor de $\mathrm{pH}$ muito baixo. Como os fertilizantes na fertirrigação por gotejo são aplicados de forma 
localizada, isso provocou maior acidificação do solo nestes tratamentos, pois promoveu maior lixiviação de íons na região do bulbo úmido, em comparação à adubação convencional na qual os fertilizantes são aplicados em maior superfície. Resultados semelhantes foram observados por Laurindo (2005) que, num estudo comparando diferentes sistemas de fertirrigação, como microaspersão e gotejo com uma e duas linhas, observou maior acidificação do solo nos tratamentos fertirrigados por gotejo com uma e duas linhas e por último a microaspersão. Ou seja, quanto menor a área de aplicação dos fertilizantes menores foram os teores de $\mathrm{Ca}$ e $\mathrm{Mg}$ e, conseqüentemente, menores valores de $\mathrm{pH}$.

$\mathrm{Na}$ Tabela 3, referente à análise de solo realizada no ano de 2005, não foi observada variação acentuada de pH entre os tratamentos, que variou entre 4,0 e 5,0. Resultados semelhantes foram observados para os anos de 2003 e 2004, razão pela qual eles não foram apresentados. Observa-se que, nas amostragens posteriores, as aplicações localizadas de calcário sob a projeção das plantas, em julho de 2002 e setembro de 2003, os valores de $\mathrm{pH}$ se mantiveram mais elevados do que no primeiro ano do experimento. Isso mostra que a calagem localizada, por adicionar grande quantidade de calcário na zona do bulbo, permitiu a correção contínua da acidificação do solo, reduzindo-se assim o impacto negativo da fertirrigação na acidificação excessiva do solo.

\section{CONCLUSÕES}

1) Os tratamentos fertirrigados, em geral, apresentaram as maiores concentrações de íons na solução do solo e, conseqüentemente, os maiores valores de condutividade elétrica.

2) Teores elevados de $\mathrm{NH}_{4}^{+}$e $\mathrm{NO}_{3}^{-}$foram observados na solução do solo nos tratamentos fertirrigados, mostrando que existem limitações para a nitrificação e grande potencial de perdas de nitrogênio por lixiviação.

3) A movimentação de P para camadas mais profundas do perfil do solo foi facilitada pela fertirrigação.

4) A acidificação do solo foi mais intensa nos tratamentos fertirrigados, sendo que aplicação localizada de calcário sob a projeção da copa permitiu a correção contínua da acidez, o que reduziu o impacto negativo da fertirrigação sobre a acidez do perfil do solo.

\section{AGRADECIMENTOS}

À fundação de Amparo à Pesquisa do Estado de São Paulo (FAPESP), pelo auxílio financeiro para a realização do experimento e pela concessão de bolsa, e às empresas Branco Peres Cítrus, NetafimBrasil-Sistemas e Equipamentos de Irrigação e FORBB- Serviços de Consultoria em Irrigação, pela contribuição e participação na realização deste projeto de Pesquisa.

\section{REFERÊNCIAS}

COSTA , E. E.; FRANÇA, G.E.; ALVES, V.M.C. Aplicação de fertilizantes via água de irrigação. Informe Agropecuário. Belo Horizonte, Belo Horizonte, v.12, p. 63-68, 1986.

DUENHAS, L. H.; VILLAS BÔAS, R. L.; SOUZA, C. M. P.; RAGOSO, R. A.; BULL, L.T. Fertirrigação com diferentes doses de NPK e seus efeitos sobre a produção e qualidade de frutos de laranja (Citrus sinensis O.) 'Valência'. Revista Brasileira de Fruticultura, Jaboticabal, v. 24, n. 1, p. 214-218, 2002.

FOUCHE, P.S.; BESTER, D.H. The influence of water soluble fertilizers on the nutrition and producitivity of Navel orange trees under microjetirrigation. Citrus and Subtropical Fruit Journal, Parklands, v. 626, p. 8-10, 1986.

LAURINDO, V. T. Interferência da fertirrigação, de lâminas e modos de aplicação localizada de água nos atributos químicos do solo e na produtividade da laranja Valência. 2005. 100f. Dissertação (Mestrado em Produção Vegetal) - Faculdade de Ciências Agrárias e Veterinárias, Universidade Estadual Paulista , Jaboticabal, 2005.

MINITAB. User's guide 2: Data analysis and quality tools. Release 13. State College, 752p., 2000.

PAPADOPOULOS, I. Fertirrigação: situação atual e perspectivas para o futuro. In: FOLLEGATTI, M. V. (Ed.). Fertirrigação: cítrus, flores, hortaliças. Guaíba: Agropecuária, 1999. p.11-169.

QUAGGIO, J.A. Acidez e calagem em solos tropicais. Campinas: Instituto Agronômico, 2000, 111p.

RAIJ, B. van. Fertilidade do solo e adubação. Piracicaba: Editora Agronômica Ceres, 1991.343p.

RAIJ, B. van.; ANDRADE, J.C.; CANTARELLA, H.; QUAGGIO, J.A. Análise química para avaliação da fertilidade de solos tropicais. Campinas: Instituto Agronômico, 2001. 285p.

RAUSCHKOLB, R.S.; ROLSTON, D.E.; MILLER, R.J.; CARLTON, A.B.; BAURAU, R.G. Phosphorus fertilization with drip irrigation. Soil Science Society of America Journal, Madison, v. 40, p. 68-72, 1976.

SOUZA, F.V.; FOLLEGATTI, M.V.; FRIZZONE, J.A.; CORRÊA, R.A.L.; ALENCAR C.M. Distribuição de fertilizantes em um sistema de fertirrigação por gotejamento. Revista Brasileira de Engenharia Agrícola e Ambiental, Campina Grande, v.7, n. 1, p.186-189, 2003.

TSAI, S.M.; BARAIBAR, A.V.L.; ROMANI, V.L.M. Efeitos de fatores do solo. In: CARDOSO, E.J.B.N.; TSAI, S.M.; NEVES, M.C.P (Ed.). Microbiologia do solo. Campinas: Instituto Agronômico, 1992. p. 58-74.

VILLAS BÔAS, R. L.; BÜLL, L.T.; FERNANDES, D.M. Fertilizantes em fertirrigação. In: FOLLEGATTI, M. V. (Ed.). Fertirrigação: citros, flores, hortaliças. Guaíba: Agropecuária, 1999. p. 293319.

VILLAS BOAS, R.L.; OLIVEIRA, M.V.A.M.; MOTA, P.R.D.; BETTINI, M.O. Agricultura fertirrigada avança no Brasil. In: AGRIANUAL 2005: anuário da agricultura brasileira. São Paulo: FNP Consultoria e Agroinformativos, 2005. p.54-57. 